\title{
Sequential design of multi-overlapping controllers for longitudinal multi-overlapping systems
}

\author{
Francisco Palacios-Quiñonero ${ }^{\mathrm{a}}$, José Rodellar ${ }^{\mathrm{b}}$, \\ Josep M. Rossell ${ }^{\text {a }}$ \\ ${ }^{a}$ Department of Applied Mathematics III, Universitat Politècnica de Catalunya \\ (UPC), 08240-Manresa, Spain \\ ${ }^{\mathrm{b}}$ Department of Applied Mathematics III, Universitat Politècnica de Catalunya \\ (UPC), Campus Nord, C-2, 08034-Barcelona
}

\begin{abstract}
In this paper a strategy to design multi-overlapping controllers for systems with longitudinal multi-overlapping structure is presented. The design process uses the inclusion principle to carry out a multi-step expansion that allows the decentralized design of a sequence of expanded local controllers. These expanded local controllers are subsequently contracted to produce a multi-overlapping controller that can be implemented in the original system. To illustrate the proposed methodology, the response of a four-story building under a seismic excitation is considered.
\end{abstract}

Key words: Inclusion principle, multi-overlapping decomposition, information structure constraints, decentralized control, building structures.

\section{Introduction}

Dimensionality, uncertainty, and information constraints are fundamental characteristics of system complexity [9]. When a complex system can be decomposed into disjoint subsystems, a set of local controllers may be independently

Email addresses: francisco.palacios@upc.edu (Francisco Palacios-Quiñonero), jose.rodellar@upc.edu (José Rodellar), josep.maria.rossell@upc.edu (Josep M. Rossell).

1 This work was supported in part by the Spanish Committee for Science and Technology (CICYT) under Grant DPI2008-06699-C02-02 and DPI2008-06564-C0202 
obtained to design a decentralized controller. Design and operation of local controllers requires lower-dimension computation, minimizes the information exchange, and increases the global robustness by reducing the effect of perturbations and failures on interconnections. However, all these potential benefits are severely attenuated by the fact that systems encountered in practical applications rarely admit a perfect disjoint decomposition.

Overlapping decomposition overcomes this serious drawback by allowing the subsystems to overlap; that is, the requirement of strict disjoint decomposition is relaxed permitting a restricted sharing of states, inputs, and outputs among the subsystems. For systems admitting an overlapping decomposition, the Inclusion Principle allows the design of overlapping controllers which are in accordance with the system structure, and which partially maintains the features of decentralized controllers: low dimensionality, reduced information exchange, and robustness with respect to interconnection degradation. The basic idea is to expand the original system into a bigger (virtual) one, where the overlapped subsystems appear as disjoint. After that, local controllers can be independently designed using standard methods for the disjoint expanded subsystems. The decentralized controller for the expanded system so obtained is then contracted back to the original system for implementation. This approach has proven to be useful in a variety of complex control problems appearing in very different fields, such as economic systems, electric power systems, automated highway systems, civil engineering, large space structures, applied mathematics, formation flight of aerial vehicles, and communication networks. Theoretical results about the Inclusion Principle include stochastic, hereditary, discrete-time, nonlinear and hybrid systems. It has to be noted, however, that the majority of theoretical results and applications have been formulated for the simple case of two overlapping subsystems, while most of the problems appearing in the context of large scale and complex systems lead naturally to the consideration of multi-overlapping structures.

The generalization from a simple overlapping to a multi-overlapping approach is by no means straightforward. For three overlapping subsystems, three different overlapping topologies can be considered: longitudinal, loop, and radial. These basic multi-overlapping structures are illustrated in Fig. 1. The circles in the diagrams can be seen as physical subsystems, the arrows indicate state, input, or output interaction, while the dotted ellipses represent the overlapping subsystems. Obviously, in the general case of $n$ overlapping subsystems, very complex multi-overlapping structures may appear. A very interesting approach to multi-overlapping controller design for general multi-overlapping structures can be found in [5].

In this paper, we present a sequential procedure to design multi-overlapped controllers for systems admitting a longitudinal multi-overlapping decomposition. In the usual approach to multi-overlapping decomposition, a generalized 


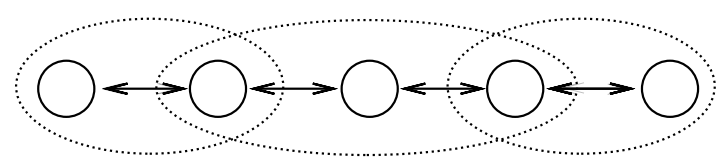

(a) Longitudinal

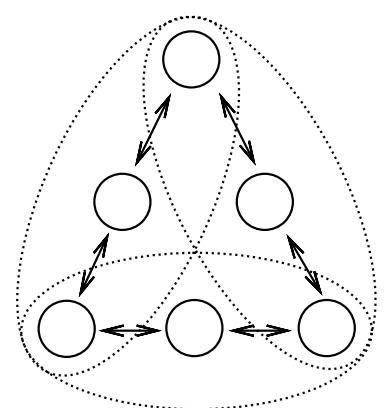

(b) Loop

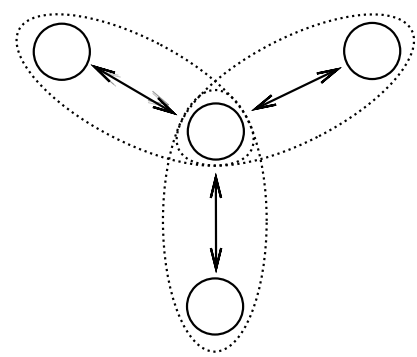

(c) Radial

Fig. 1. Three-subsystem multi-overlapping decompositions.

version of the Inclusion Principle is normally used. The expansion, decoupling, and contraction processes are performed in a single step using generalized forms of the expansion and complementary matrices, whose structure depend on the structure of the particular decomposition considered. In the presented sequential strategy, a chain of $(r+1)$ subsystems with longitudinal multioverlapping $\mathbf{S}=\left(\mathbf{S}^{(1)}, \ldots, \mathbf{S}^{(r+1)}\right)$ is breaking into $(r+1)$ decoupled expanded subsystems $\tilde{\mathbf{S}}^{(1)}, \ldots, \tilde{\mathbf{S}}^{(r+1)}$, in $r$ steps. In the $j$ th step, a simple overlapping decomposition is performed to produce a decoupled extended subsystem $\widetilde{\mathbf{S}}^{(j+1)}$ and a shorter expanded chain $\overline{\mathbf{S}}^{(j)}=\left(\mathbf{S}^{(1)}, \ldots, \mathbf{S}^{(r+1)}\right)$. Controllers for the decoupled extended systems can be independently designed and, when the expansion is complete, sequentially contracted to obtain a multi-overlapping controller for the original system. The fact that only simple two-subsystem overlapping decompositions are needed adds a remarkable theoretical and computational simplicity to the proposed sequential approach.

The present work is part of a research project aimed at designing wireless control systems to mitigate the vibrational response of tall buildings under seismic excitations. This problem has some specific characteristics that fit specially well into the theoretical framework of longitudinal multi-overlapping decomposition: (i) Structure. A tall building can be seen as a chain of interconnected stories. Interactions only occur between adjacent stories. (ii) High dimensionality. In real applications, buildings with more than a hundred stories can be considered. In these cases, even simplified models will involve hundreds of states. (iii) Information constraints. Using wireless communications, instead of the classical coaxial wiring, can critically reduce the installation and maintenance costs. It also adds flexibility to the control system, allowing the implementation of new control schemes without costly wiring modifications. 
However, to improve the robustness of communications and to achieve higher sampling frequencies, the controllers should operate using local information provided by neighboring sensors.

Accordingly, a simplified model of a four-story building has been chosen to illustrate the application of the proposed sequential methodology in a practical context. For this model, multi-overlapping controllers for two different actuation schemes have been designed and compared with the corresponding centralized controllers. The 1940 El Centro NS earthquake has been used as a ground acceleration input in the simulations.

The organization of the paper is as follows. Section 2 gives necessary background results about the inclusion principle and the design of overlapping controllers. Section 3 presents the sequential multi-step procedure to compute multi-overlapping controllers. In Section 4, a simplified model of a four-story building is presented, and the centralized and multi-overlapping controllers are computed. Finally, in Section 5, numerical simulations of the response to the El Centro earthquake are used to compare the performance of the obtained controllers.

\section{Background Results}

In this section, some basic definitions and results related to the Inclusion Principle and the design of overlapping controllers in the case of two overlapping subsystems are briefly presented. A rigorous treatment can be found in [1], $[2],[5],[6],[7],[8],[9]$.

\subsection{Inclusion Principle}

Consider a pair of linear systems

$$
\mathbf{S}: \dot{x}(t)=A x(t)+B u(t), \quad \tilde{\mathbf{S}}: \dot{\tilde{x}}(t)=\tilde{A} \tilde{x}(t)+\tilde{B} \tilde{u}(t),
$$

where $x(t) \in \mathbb{R}^{\mathbf{n}}, u(t) \in \mathbb{R}^{\mathbf{m}}$ represent the state and input of $\mathbf{S}$ at time $t \in \mathbb{R}^{+}$, and $\tilde{x}(t) \in \mathbb{R}^{\tilde{\mathbf{n}}}, \tilde{u}(t) \in \mathbb{R}^{\tilde{\mathbf{m}}}$ are the state and input of $\tilde{\mathbf{S}}$. $A, B, \tilde{A}$, and $\tilde{B}$, are constant matrices of dimensions $n \times n, n \times m, \tilde{n} \times \tilde{n}, \tilde{n} \times \tilde{m}$, respectively. The dimensions of the state and input vectors $x, u$ of $\mathbf{S}$ are supposed to be smaller than the dimensions of $\tilde{x}, \tilde{u}$ of $\tilde{\mathbf{S}}$. The state behavior of $\mathbf{S}$ for the input $u(t)$ and the initial state $x(0)=x_{0}$ is denoted by $x\left(t ; x_{0}, u\right)$. A similar notation $\tilde{x}\left(t ; \tilde{x}_{0}, \tilde{u}\right)$ is used for the state behavior of the system $\tilde{\mathbf{S}}$. 
Let us consider the following transformations:

$$
V: \mathbb{R}^{\mathbf{n}} \longrightarrow \mathbb{R}^{\tilde{\mathbf{n}}}, \quad U: \mathbb{R}^{\tilde{\mathbf{n}}} \longrightarrow \mathbb{R}^{\mathbf{n}}, \quad R: \mathbb{R}^{\mathbf{m}} \longrightarrow \mathbb{R}^{\tilde{\mathbf{m}}}, \quad Q: \mathbb{R}^{\tilde{\mathbf{m}}} \longrightarrow \mathbb{R}^{\mathbf{m}}
$$

where $\operatorname{rank}(V)=n, \operatorname{rank}(R)=m$ and such that $U V=I_{n}, Q R=I_{m}$, where $I_{n}$ and $I_{m}$ are the identity matrices of indicated dimensions.

Definition 1 (Inclusion Principle) A system $\tilde{\mathbf{S}}$ is an expansion of the system $\mathbf{S}$ if there exist transformations $(U, V, R, S)$ as in (2) such that, for any initial state $x_{0} \in \mathbb{R}^{\mathbf{n}}$ and any input $u(t) \in \mathbb{R}^{\mathbf{m}}$, if $\tilde{x}_{0}=V x_{0}$ and $\tilde{u}(t)=R u(t)$ then $x\left(t ; x_{0}, u\right)=U \tilde{x}\left(t ; V x_{0}, R u\right)$ for all $t \geq 0$.

Definition 2 (Contractibility) Suppose that $\tilde{\mathbf{S}}$ is an expansion of the system $\mathbf{S}$. Then, a control law $\tilde{u}(t)=-\tilde{K} \tilde{x}(t)$ for $\tilde{\mathbf{S}}$ is contractible to the control law $u(t)=-K x(t)$ for $\mathbf{S}$ if there exist transformations as in (2) such that, for any initial state $x_{0} \in \mathbb{R}^{\mathbf{n}}$ and any input $u(t) \in \mathbb{R}^{\mathrm{m}}$, if $\tilde{x}_{0}=V x_{0}$ and $\tilde{u}(t)=R u(t)$ then $K x\left(t ; x_{0}, u\right)=Q \tilde{K} \tilde{x}\left(t ; V x_{0}, R u\right)$ for all $t \geq 0$.

Under the transformations (2), both systems $\mathbf{S}$ and $\tilde{\mathbf{S}}$ can be related as $\tilde{A}=V A U+M, \tilde{B}=V B Q+N$, where $M$ and $N$ are complementary matrices of appropriate dimensions. In terms of complementary matrices, the inclusion principle and the contractibility conditions can be established in the following way.

Proposition 3 A system $\tilde{\mathbf{S}}$ is an expansion of the system $\mathbf{S}$ if and only if $U M^{i} V=0, U M^{i-1} N R=0$, for $i=1, \ldots, \tilde{n}$.

Proposition 4 Suppose that $\tilde{\mathbf{S}}$ is an expansion of the system $\mathbf{S}$. Then, a control law $\tilde{u}(t)=-\tilde{K} \tilde{x}(t)$ for $\tilde{\mathbf{S}}$ is contractible to the control law $u(t)=-K x(t)$ for $\mathbf{S}$ if and only if $Q \tilde{K} V=K, Q \tilde{K} M^{i} V=0, Q \tilde{K} M^{i-1} N R=0$, for $i=1, \ldots, \tilde{n}$.

For $\tilde{\mathbf{S}}$ to be an expansion of $\mathbf{S}$, a proper choice of $M$ and $N$ is required. It is well known that, by adding complementary matrices, different expanded systems $\tilde{\mathbf{S}}$ can be obtained with some degree of freedom in the expansion-contraction process [3], [4]. There are two special cases of expansions, which are called restrictions and aggregations, [9]. In this paper we will use a restriction to expand the original system. The structures of the complementary matrices $M$ and $N$ are provided by the following proposition.

Proposition 5 A system $\tilde{\mathbf{S}}$ is a restriction of the system $\mathbf{S}$ if and only if $M V=0, N R=0$. Then, the matrices $M$ and $N$ have the form

$$
M=\left[\begin{array}{llll}
0 & M_{12} & -M_{12} & 0 \\
0 & M_{22} & -M_{22} & 0 \\
0 & M_{32} & -M_{32} & 0 \\
0 & M_{42} & -M_{42} & 0
\end{array}\right], \quad N=\left[\begin{array}{llll}
0 & N_{12} & -N_{12} & 0 \\
0 & N_{22} & -N_{22} & 0 \\
0 & N_{32} & -N_{32} & 0 \\
0 & N_{42} & -N_{42} & 0
\end{array}\right]
$$


If a restriction is used, any control law designed in the expanded space can be contracted to the initial one. Using this property, controllers designed in the expanded space $\tilde{\mathbf{S}}$ can be subsequently contracted and implemented in the initial space $\mathbf{S}$.

\subsection{Controller design for two overlapping subsystems}

Let us suppose that the system

$$
\mathbf{S}: \quad \dot{x}(t)=A x(t)+B u(t)
$$

admits an overlapping decomposition as shown in Fig. 2, where the circles represent subsystems, the arrows indicate state, input, or output direct interaction, and the dotted ellipses represent the overlapping subsystems. As is well represented in the diagram, the key feature of overlapping decomposition is that no direct interaction exists between $\mathbf{S}_{1}$ and $\mathbf{S}_{3}$. In terms of the system

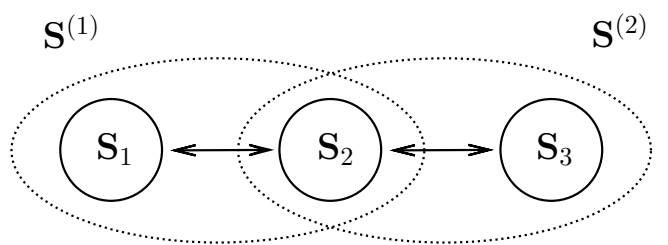

Fig. 2. Two-subsystem overlapping decomposition.

matrices, this property means that matrices $A$ and $B$ corresponding to the system (4) will present a block tridiagonal structure

$$
A=\left[\begin{array}{cc:c}
A_{11} & A_{12} & 0 \\
\hdashline A_{21} & A_{22} & A_{23} \\
\hdashline 0 & A_{32} & A_{33}
\end{array}\right], \quad B=\left[\begin{array}{cc:c}
B_{11} & B_{12} & 0 \\
\hdashline B_{21} & B_{22} & B_{23} \\
\hdashline 0 & B_{32} & B_{33}
\end{array}\right]
$$

where $A_{i i}, B_{i j}$, for $i, j=1,2,3$, are $n_{i} \times n_{i}, n_{i} \times m_{j}$ dimensional matrices, respectively. Subsystems $\mathbf{S}_{i}$ are described by

$$
\mathbf{S}_{i}: \quad \dot{x}_{i}(t)=A_{i i} x_{i}(t)+B_{i i} u_{i}(t), \quad i=1,2,3,
$$

the partition of the state $x=\left(x_{1}^{T}, x_{2}^{T}, x_{3}^{T}\right)^{T}$ has components of respective dimensions $n_{1}, n_{2}, n_{3}$, satisfying $n_{1}+n_{2}+n_{3}=n$; and the partition of $u=\left(u_{1}^{T}, u_{2}^{T}, u_{3}^{T}\right)^{T}$ has components of dimensions $m_{1}, m_{2}, m_{3}$, such that $m_{1}+m_{2}+m_{3}=m$.

The design of overlapping controllers using the inclusion principle has two main features: (i) Structure. The resulting controller fits in with the system structure. More precisely, a control law $u(t)=-K_{o} x(t)$ with a block tridiagonal 
gain matrix

$$
K_{o}=\left[\begin{array}{cc:c}
K_{11} & K_{12} & 0 \\
K_{21} & -K_{22} & K_{23} \\
\hdashline 0 & K_{32} & K_{33}
\end{array}\right]
$$

is obtained. (ii) Lower dimensionality. The gain matrix $K_{o}$ is computed from two lower-dimension controllers which are independently designed.

The controller design starts with the definition of the expansion transformations

$$
V=\left[\begin{array}{ccc}
I_{n_{1}} & 0 & 0 \\
0 & I_{n_{2}} & 0 \\
0 & I_{n_{2}} & 0 \\
0 & 0 & I_{n_{3}}
\end{array}\right], \quad R=\left[\begin{array}{ccc}
I_{m_{1}} & 0 & 0 \\
0 & I_{m_{2}} & 0 \\
0 & I_{m_{2}} & 0 \\
0 & 0 & I_{m_{3}}
\end{array}\right]
$$

together with their corresponding pseudoinverse contractions

$$
U=\left(V^{T} V\right)^{-1} V^{T}=\left[\begin{array}{cccc}
I_{n_{1}} & 0 & 0 & 0 \\
0 & \frac{1}{2} I_{n_{2}} & \frac{1}{2} I_{n_{2}} & 0 \\
0 & 0 & 0 & I_{n_{3}}
\end{array}\right], \quad Q=\left(R^{T} R\right)^{-1} R^{T}=\left[\begin{array}{cccc}
I_{m_{1}} & 0 & 0 & 0 \\
0 & \frac{1}{2} I_{m_{2}} \frac{1}{2} I_{m_{2}} & 0 \\
0 & 0 & 0 & I_{m_{3}}
\end{array}\right] \text {. }
$$

The expanded matrices $\bar{A}=V A U, \bar{B}=V B Q$, have the form

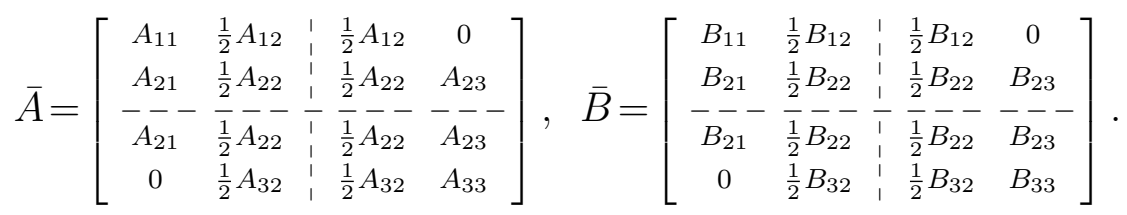

In order to get an almost-decoupled expanded system, we add complementary matrices as indicated in Proposition 5,

$$
M=\left[\begin{array}{rrrr}
0 & \frac{1}{2} A_{12} & -\frac{1}{2} A_{12} & 0 \\
0 & \frac{1}{2} A_{22} & -\frac{1}{2} A_{22} & 0 \\
0 & -\frac{1}{2} A_{22} & \frac{1}{2} A_{22} & 0 \\
0 & -\frac{1}{2} A_{32} & \frac{1}{2} A_{32} & 0
\end{array}\right], \quad N=\left[\begin{array}{rrrr}
0 & \frac{1}{2} B_{12} & -\frac{1}{2} B_{12} & 0 \\
0 & \frac{1}{2} B_{22} & -\frac{1}{2} B_{22} & 0 \\
0 & -\frac{1}{2} B_{22} & \frac{1}{2} B_{22} & 0 \\
0 & -\frac{1}{2} B_{32} & \frac{1}{2} B_{32} & 0
\end{array}\right]
$$

resulting

$$
\begin{gathered}
\tilde{A}=V A U+M=\left[\begin{array}{cc}
\tilde{A}_{11} & \tilde{A}_{12} \\
\tilde{A}_{21} & \tilde{A}_{22}
\end{array}\right]=\left[\begin{array}{cc:cc}
A_{11} & A_{12} & 0 & 0 \\
A_{21} & A_{22} & 0 & A_{23} \\
\hdashline A_{21} & 0 & A_{22} & A_{23} \\
0 & 0 & A_{32} & A_{33}
\end{array}\right], \\
\tilde{B}=V B Q+N=\left[\begin{array}{cc}
\tilde{B}_{11} & \tilde{B}_{12} \\
\tilde{B}_{21} & \tilde{B}_{22}
\end{array}\right]=\left[\begin{array}{cc:cc}
B_{11} & B_{12} & 0 & 0 \\
B_{21} & B_{22} & 0 & B_{23} \\
\hdashline B_{21} & 0 & B_{22} & B_{23} \\
0 & 0 & B_{32} & B_{33}
\end{array}\right] .
\end{gathered}
$$


The expanded system

$$
\tilde{\mathbf{S}}: \quad \dot{\tilde{x}}(t)=\tilde{A} \tilde{x}(t)+\tilde{B} \tilde{u}(t),
$$

with state $\tilde{x}^{T}=\left(x_{1}^{T}, x_{2}^{T}, x_{2}^{T}, x_{3}^{T}\right)$, and input $\tilde{u}^{T}=\left(u_{1}^{T}, u_{2}^{T}, u_{2}^{T}, u_{3}^{T}\right)$, admits an almostdecoupled decomposition. Using the block notation given in (12) and (13), we can write

$$
\begin{array}{ll}
\tilde{\mathbf{S}}_{1}: & \dot{\tilde{x}}_{1}(t)=\tilde{A}_{11} \tilde{x}_{1}(t)+\tilde{B}_{11} \tilde{u}_{1}(t)+\tilde{A}_{12} \tilde{x}_{2}(t)+\tilde{B}_{12} \tilde{u}_{2}(t), \\
\tilde{\mathbf{S}}_{2}: & \dot{\tilde{x}}_{2}(t)=\tilde{A}_{22} \tilde{x}_{2}(t)+\tilde{B}_{22} \tilde{u}_{2}(t)+\tilde{A}_{21} \tilde{x}_{1}(t)+\tilde{B}_{21} \tilde{u}_{1}(t),
\end{array}
$$

where $\tilde{x}_{1}^{T}=\left(x_{1}^{T}, x_{2}^{T}\right), \tilde{u}_{1}^{T}=\left(u_{1}^{T}, u_{2}^{T}\right), \tilde{x}_{2}^{T}=\left(x_{2}^{T}, x_{3}^{T}\right)$, and $\tilde{u}_{2}^{T}=\left(u_{2}^{T}, u_{3}^{T}\right)$. Note that the interconnection matrices $\tilde{A}_{i j}, \tilde{B}_{i j}, i, j=1,2, i \neq j$, can have at most one non-zero block. By removing the weak interconnection terms, two decoupled expanded subsystems result

$$
\begin{array}{ll}
\tilde{\mathbf{S}}_{\mathbf{D}}^{(1)}: & \dot{\tilde{x}}_{1}(t)=\tilde{A}_{11} \tilde{x}_{1}(t)+\tilde{B}_{11} \tilde{u}_{1}(t), \\
\tilde{\mathbf{S}}_{\mathbf{D}}^{(2)}: & \dot{\tilde{x}}_{2}(t)=\tilde{A}_{22} \tilde{x}_{2}(t)+\tilde{B}_{22} \tilde{u}_{2}(t),
\end{array}
$$

which define a decoupled expanded system

$$
\tilde{\mathbf{S}}_{\mathbf{D}}: \quad \dot{\tilde{x}}(t)=\tilde{A}_{D} \tilde{x}(t)+\tilde{B}_{D} \tilde{u}(t),
$$

with $\tilde{A}_{D}=\operatorname{diag}\left\{\tilde{A}_{11}, \tilde{A}_{22}\right\}$ and $\tilde{B}_{D}=\operatorname{diag}\left\{\tilde{B}_{11}, \tilde{B}_{22}\right\}$.

At this point, we have to design a decentralized controller for $\tilde{\mathbf{S}}_{\mathbf{D}}$. This can be done by independently computing local controllers for $\tilde{\mathbf{S}}_{\mathbf{D}}^{(1)}$ and $\tilde{\mathbf{S}}_{\mathbf{D}}^{(2)}$. For simplicity, all the controllers in this paper will be designed as optimal LQR controllers. However, it is clear that other control strategies could have been used. For the expanded decoupled subsystems $\tilde{\mathbf{S}}_{\mathrm{D}}^{(1)}$ and $\tilde{\mathbf{S}}_{\mathrm{D}}^{(2)}$, let us consider the local quadratic cost functions

$$
\begin{aligned}
& \tilde{J}_{D}^{(1)}\left(\tilde{x}_{10}, \tilde{u}_{1}(t)\right)=\int_{0}^{\infty}\left[\tilde{x}_{1}^{T}(t) \tilde{Q}_{1}^{*} \tilde{x}_{1}(t)+\tilde{u}_{1}^{T}(t) \tilde{R}_{1}^{*} \tilde{u}_{1}(t)\right] d t \\
& \tilde{J}_{D}^{(2)}\left(\tilde{x}_{20}, \tilde{u}_{2}(t)\right)=\int_{0}^{\infty}\left[\tilde{x}_{2}^{T}(t) \tilde{Q}_{2}^{*} \tilde{x}_{2}(t)+\tilde{u}_{2}^{T}(t) \tilde{R}_{2}^{*} \tilde{u}_{2}(t)\right] d t
\end{aligned}
$$

where $\tilde{x}_{10}$ and $\tilde{x}_{20}$ are the initial states of $\tilde{\mathbf{S}}_{\mathbf{D}}^{(1)}$ and $\tilde{\mathbf{S}}_{\mathbf{D}}^{(2)}$, respectively, and $\tilde{Q}_{1}^{*}$, $\tilde{Q}_{2}^{*}, \tilde{R}_{1}^{*}$ and $\tilde{R}_{2}^{*}$ are appropriate expanded matrices. The gain matrices for the control laws that minimize the cost functions (18)

$$
\tilde{u}_{1}(t)=-\tilde{K}_{1} \tilde{x}_{1}(t), \quad \tilde{u}_{2}(t)=-\tilde{K}_{2} \tilde{x}_{2}(t),
$$

can be independently computed as

$$
\tilde{K}_{1}=\left[\tilde{R}_{1}^{*}\right]^{-1} \tilde{B}_{1}^{T} \tilde{P}_{1}, \quad \tilde{K}_{2}=\left[\tilde{R}_{2}^{*}\right]^{-1} \tilde{B}_{2}^{T} \tilde{P}_{2},
$$


where $\tilde{P}_{1}$ and $\tilde{P}_{2}$ are the solutions of the corresponding Riccati equations. In the decoupled expanded system $\tilde{\mathbf{S}}_{\mathbf{D}}$, the gain matrix of the controller $\tilde{u}(t)=-\tilde{K}_{D} \tilde{x}(t)$ which minimizes the cost function

$$
\tilde{J}_{D}\left(\tilde{x}_{0}, \tilde{u}(t)\right)=\int_{0}^{\infty}\left[\tilde{x}^{T}(t) \tilde{Q}_{D}^{*} \tilde{x}(t)+\tilde{u}^{T}(t) \tilde{R}_{D}^{*} \tilde{u}(t)\right] d t
$$

with $\tilde{Q}_{D}^{*}=\operatorname{diag}\left\{\tilde{Q}_{1}^{*}, \tilde{Q}_{2}^{*}\right\}$ and $\tilde{R}_{D}^{*}=\operatorname{diag}\left\{\tilde{R}_{1}^{*}, \tilde{R}_{2}^{*}\right\}$, can be written as a block diagonal gain matrix $\tilde{K}_{D}^{*}=\operatorname{diag}\left\{\tilde{K}_{1}^{*}, \tilde{K}_{2}^{*}\right\}$. Finally, the controller $\tilde{u}_{D}(t)=-\tilde{K}_{D} \tilde{x}(t)$ is contracted to an overlapping controller $u(t)=-K_{o} x(t)$ to be implemented into the original system $\mathbf{S}$. The contracted gain matrix is computed as

$$
K_{o}=Q \tilde{K}_{D} V
$$

and it has the desired block tridiagonal structure shown in (7).

\section{Sequential decomposition}

Let us consider a system

$$
\mathbf{S}: \dot{x}(t)=A x(t)+B u(t)
$$

formed by $(r+1)$ overlapping subsystems

$$
\mathbf{S}^{(j)}: \dot{x}^{(j)}(t)=A^{(j)} x^{(j)}(t)+B^{(j)} u^{(j)}(t), \quad j=1, \ldots, r+1 .
$$

The system matrix $A$ has the block structure shown in Fig. 3 with $(r+1)$ main blocks $A^{(j)}$ and $r$ overlapping sub-blocks $O_{A}^{(j)}$. The input matrix $B$ presents
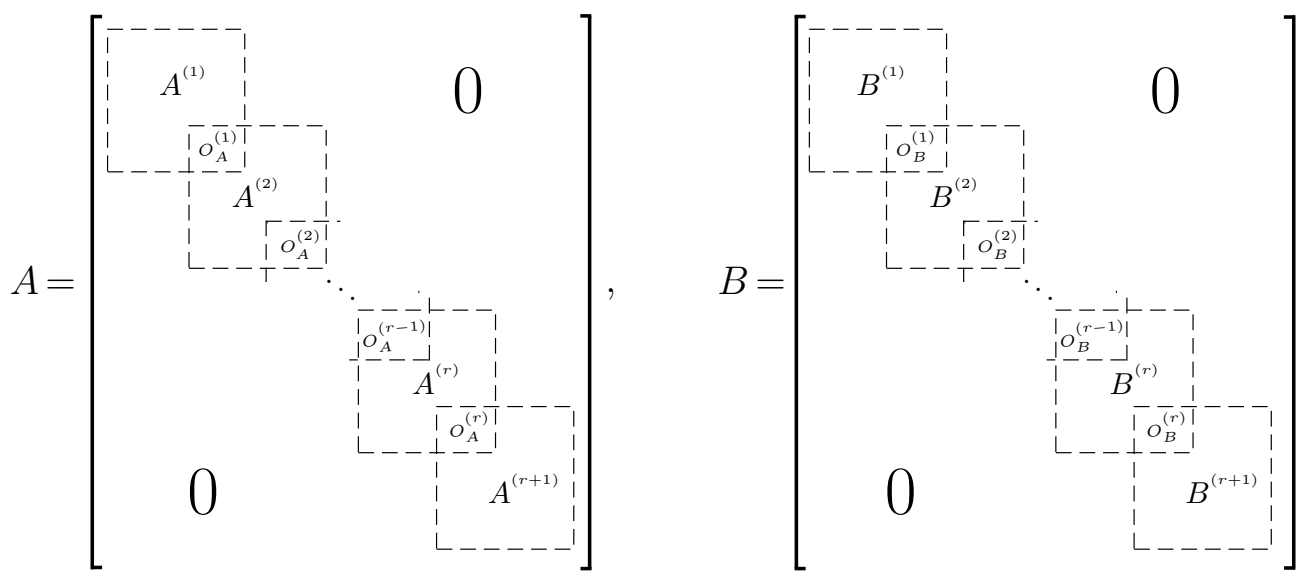

Fig. 3. Block structures of matrices $A$ and $B$.

an analogous structure. Broadly speaking, the basic idea is to consider the first $r$ subsystems as a single subsystem which overlaps with $\mathbf{S}^{(r+1)}$. Then, 
the standard theory for two overlapped systems may be applied to obtain an expanded space where a local controller can be designed for the expanded subsystem $\tilde{\mathbf{S}}^{(r+1)}$. This step also produces another expanded system with only $r$ overlapped subsystems. After $r$ expansion steps, two non-overlapped expanded subsystems result and the design of $(r+1)$ expanded local controllers can be completed. Finally, a sequence of $r$ contraction steps on the expanded local controllers yields an overlapping global controller for the initial system. For the subsystem $\mathbf{S}^{(j)}$, the state and the input dimensions are respectively denoted by $d_{x}^{(j)}$ and $d_{u}^{(j)} ; A^{(j)}$ is a square matrix of dimension $d_{x}^{(j)}$, and $B^{(j)}$ is a $d_{x}^{(j)} \times d_{u}^{(j)}$ matrix. As for the overlapping blocks, $O_{A}^{(j)}$ is a square matrix of dimension $o_{x}^{(j)}$, and $O_{B}^{(j)}$ is a $o_{x}^{(j)} \times o_{u}^{(j)}$ matrix. Note that some of the numbers $o_{u}^{(j)}$ may be zero. This is the case when there is no control overlapping between subsystems $\mathbf{S}^{(j)}$ and $\mathbf{S}^{(j+1)}$. For $j=1, \ldots, r$, we also define the numbers

$$
\left\{\begin{array} { l } 
{ n _ { 1 } ^ { ( j ) } = \sum _ { i = 1 } ^ { j } ( d _ { x } ^ { ( i ) } - o _ { x } ^ { ( i ) } ) } \\
{ n _ { 2 } ^ { ( j ) } = o _ { x } ^ { ( j ) } } \\
{ n _ { 3 } ^ { ( j ) } = d _ { x } ^ { ( j + 1 ) } - o _ { x } ^ { ( j ) } }
\end{array} \quad \left\{\begin{array}{l}
m_{1}^{(j)}=\sum_{i=1}^{j}\left(d_{u}^{(i)}-o_{u}^{(i)}\right) \\
m_{2}^{(j)}=o_{u}^{(j)} \\
m_{3}^{(j)}=d_{u}^{(j+1)}-o_{u}^{(j)}
\end{array}\right.\right.
$$

We start with the pair $P_{1}^{(r+1)}=(A, B)$ and consider the expansion matrices

$$
V^{(r)}=\left[\begin{array}{ccc}
I_{n_{1}(r)} & 0 & 0 \\
0 & I_{n_{2}(r)} & 0 \\
0 & I_{n_{2}(r)} & 0 \\
0 & 0 & I_{n_{3}(r)}
\end{array}\right], \quad R^{(r)}=\left[\begin{array}{ccc}
I_{m_{1}(r)} & 0 & 0 \\
0 & I_{m_{2}^{(r)}} & 0 \\
0 & I_{m_{2}^{(r)}} & 0 \\
0 & 0 & I_{m_{3}^{(r)}}
\end{array}\right]
$$

to perform an overlapping decomposition of $P_{1}^{(r+1)}$ as described in Subsection 2.2 , obtaining the decoupled pairs

$$
P_{1}^{(r)}=\left(\tilde{A}_{1}^{(r)}, \tilde{B}_{1}^{(r)}\right), \quad P_{2}^{(r+1)}=\left(\tilde{A}_{1}^{(r+1)}, \tilde{B}_{1}^{(r+1)}\right),
$$

as illustrated in Fig. 4. The pair $P_{2}^{(r+1)}$ contains no overlapping blocks and a

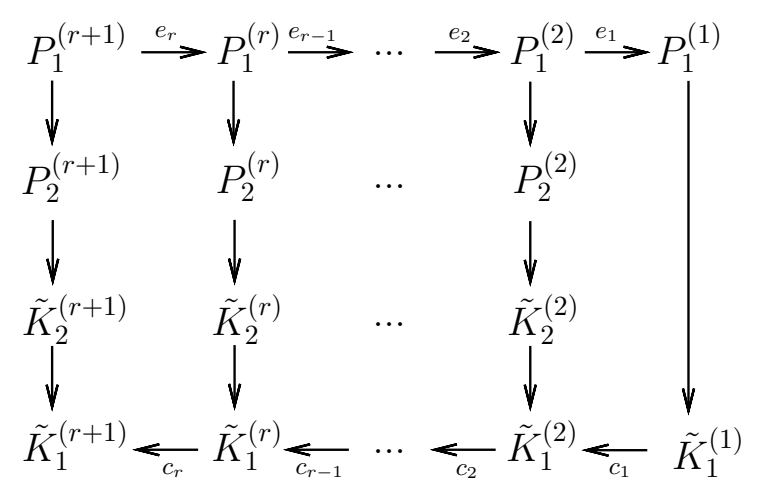

Fig. 4. Sequential overlapping decomposition scheme.

gain matrix $\tilde{K}_{2}^{(r+1)}$ can be directly designed. The other pair $P_{1}^{(r)}$ contains $r$ 
subsystems with $(r-1)$ overlapping blocks and the process needs to be repeated until a fully decoupled expansion results. More precisely, the expansion process involves $(r+1)$ pairs $P_{1}^{(j)}$ and it is carried out in $r$ steps by decreasing the index $j$ from $(r+1)$ to 1 . The expansion step $j$ starts with the pair $P_{1}^{(j+1)}$ that describes a system with $(j+1)$ subsystems and $j$ overlapping blocks and it uses expansion matrices $V^{(j)}$ and $R^{(j)}$ to yield the expanded pairs

$$
P_{1}^{(j)}=\left(\tilde{A}_{1}^{(j)}, \tilde{B}_{1}^{(j)}\right), \quad P_{2}^{(j+1)}=\left(\tilde{A}_{1}^{(j+1)}, \tilde{B}_{1}^{(j+1)}\right) .
$$

A gain matrix $\tilde{K}_{2}^{(j+1)}$ is computed for the expanded system with no overlapping blocks $P_{2}^{(j+1)}$ while the pair $P_{1}^{(j)}$, which contains $j$ subsystems and $(j-1)$ overlapping blocks, is supplied as the starting point to the next expansion step. After $r$ expansion steps, an expanded non-overlapped pair $P_{1}^{(1)}$ results, and a gain matrix $\tilde{K}_{1}^{(1)}$ is directly computed. The matrix $\bar{K}^{(1)}=\operatorname{diag}\left(\tilde{K}_{1}^{(1)}, \tilde{K}_{2}^{(2)}\right)$ can be contracted to obtain an overlapping controller $\tilde{K}_{1}^{(2)}=Q^{(1)} \bar{K}^{(1)} V^{(1)}$ for the expanded pair $P_{1}^{(2)}$. The contraction step $j$ starts with the expanded gain matrix $\tilde{K}_{1}^{(j)}$ and it uses $\tilde{K}_{2}^{(j+1)}$ to build $\bar{K}^{(j)}=\operatorname{diag}\left(\tilde{K}_{1}^{(j)}, \tilde{K}_{2}^{(j+1)}\right)$ and to compute the overlapping controller $\tilde{K}_{1}^{(j+1)}=Q^{(j)} \bar{K}^{(j)} V^{(j)}$. After $r$ contraction steps, an overlapping gain matrix $K=\tilde{K}_{1}{ }^{(r+1)}$ for the original system results.

The diagram in Fig. 4 shows schematically the whole procedure. The process starts with $P_{1}^{(r+1)}$ and the expansion progresses along the top from left to right. Note that a gain matrix $\tilde{K}_{2}^{(j+1)}$ is computed at each step; however, the expansion process needs to be completed before the first gain matrix $\tilde{K}_{1}^{(1)}$ is computed. Then, the contraction process progresses from right to left along the bottom, generating in each step the gain matrix $\tilde{K}_{1}^{(j+1)}$.

\section{Four-story building model}

To show the potential advantages of the proposed control approach and to illustrate more clearly the details of the sequential design procedure presented in the previous section, we consider the problem of reducing the response of a tall building under an earthquake excitation. More precisely, a simplified onedimensional model of a four-story building has been selected. The control goal is to reduce the inter-story drifts when the building is subjected to a ground excitation. To this end, two different actuation schemes have been considered: (a) inter-story cross-actuators, and (b) direct actuators (see Fig. 5). First, the building is considered as a whole and, for each actuation scheme, a centralized optimal LQR controller is designed. These centralized controllers will later be taken as a reference to evaluate the performance of the overlapping controllers. Secondly, the building is seen as made up of three overlapped sub- 

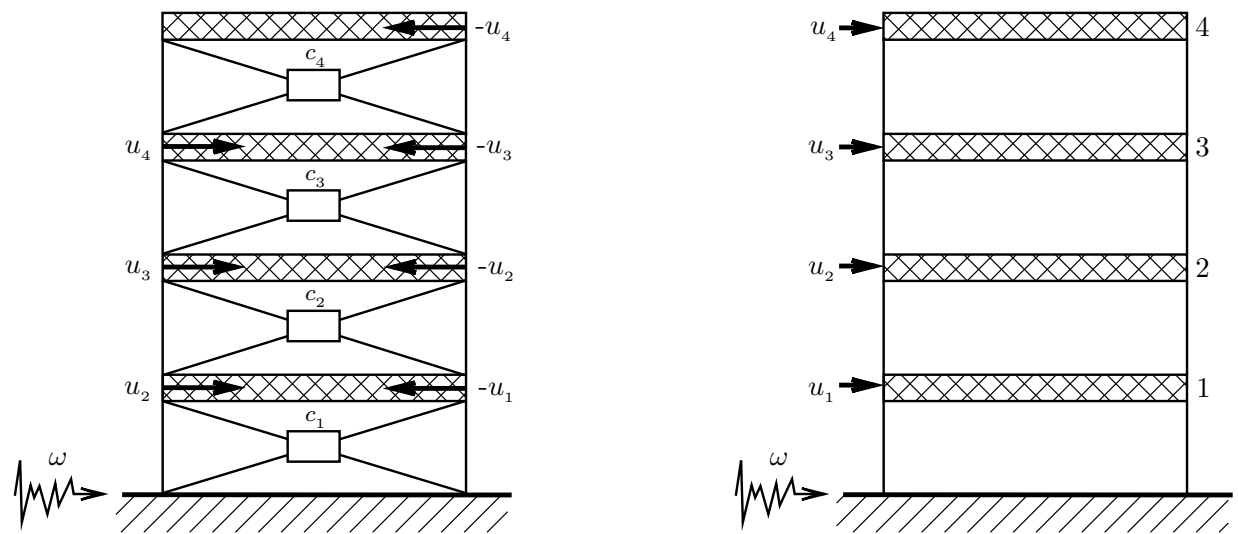

Fig. 5. Two actuation schemes for a four-story building.

systems, which include two consecutive stories, that is: $\mathbf{S}^{(1)}=[1,2], \mathbf{S}^{(2)}=[2,3]$, $\mathbf{S}^{(3)}=[3,4]$ (see Fig. 7). A sequential overlapping decomposition is then applied to this multi-overlapped system in order to obtain a multi-overlapping controller for each of the actuation schemes.

\subsection{State space model}

The building motion can be described by a second-order differential equation

$$
\mathbf{M} \ddot{q}(t)+\mathbf{C} \dot{q}(t)+\mathbf{K} q(t)=\mathbf{T}_{u} u(t)+\mathbf{T}_{w} w(t),
$$

where $q(t) \in \mathbb{R}^{4}$ is the displacement vector relative to the ground (see Fig. 6), $\mathbf{M}, \mathbf{C}, \mathbf{K}$, are the mass, damping, and stiffness matrices, respectively. The vector $u(t) \in \mathbb{R}^{4}$ is the control force, $w(t) \in \mathbb{R}$ is the ground acceleration, $\mathbf{T}_{w}$ is the excitation location matrix, and $\mathbf{T}_{u}$ is the control location matrix.

The particular values of the matrices that will be used to calculate the controllers and to carry out the simulations are the following:

$$
\begin{array}{ll}
\mathbf{M}=10^{2} \times \operatorname{diag}[3456,3456,3456,3456], & \mathbf{C}=10^{3} \times\left[\begin{array}{rrrr}
5874 & -2937 & 0 & 0 \\
-2937 & 5874 & -2937 & 0 \\
0 & -2937 & 5874 & -2937 \\
0 & 0 & -2937 & 2937
\end{array}\right], \\
\mathbf{K}=10^{5} \times\left[\begin{array}{rrrr}
6800 & -3404 & 0 & 0 \\
-3404 & 6808 & -3404 & 0 \\
0 & -3404 & 6808 & -3404 \\
0 & 0 & -3404 & 3404
\end{array}\right], & \mathbf{T}_{w}=-\mathbf{M}[1,1,1,1]^{T},
\end{array}
$$

where masses are in $k g$, damping coefficients are in $N \cdot s / m$, and stiffness coefficients are in $N / m$. As for the control location, two different matrices are considered

$$
\mathbf{T}_{u}^{(a)}=\left[\begin{array}{rrrr}
-1 & 1 & 0 & 0 \\
0 & -1 & 1 & 0 \\
0 & 0 & -1 & 1 \\
0 & 0 & 0 & -1
\end{array}\right], \quad \mathbf{T}_{u}^{(b)}=\left[\begin{array}{llll}
1 & 0 & 0 & 0 \\
0 & 1 & 0 & 0 \\
0 & 0 & 1 & 0 \\
0 & 0 & 0 & 1
\end{array}\right]
$$


corresponding, respectively, to the actuation schemes (a) and (b) shown in Fig. 5.

From the second-order model (29), a first-order state-space model can be derived

$$
\mathbf{S}: \quad \dot{x}(t)=A x(t)+B^{(j)} u(t)+E w(t),
$$

where the state vector $x(t) \in \mathbb{R}^{8}$ contains the inter-story drifts and velocities arranged in increasing order (see Fig. 6), that is,

$x(t)=\left[q_{1}, \dot{q}_{1},\left(q_{2}-q_{1}\right),\left(\dot{q}_{2}-\dot{q}_{1}\right),\left(q_{3}-q_{2}\right),\left(\dot{q}_{3}-\dot{q}_{2}\right),\left(q_{4}-q_{3}\right),\left(\dot{q}_{4}-\dot{q}_{3}\right)\right]^{T}$.
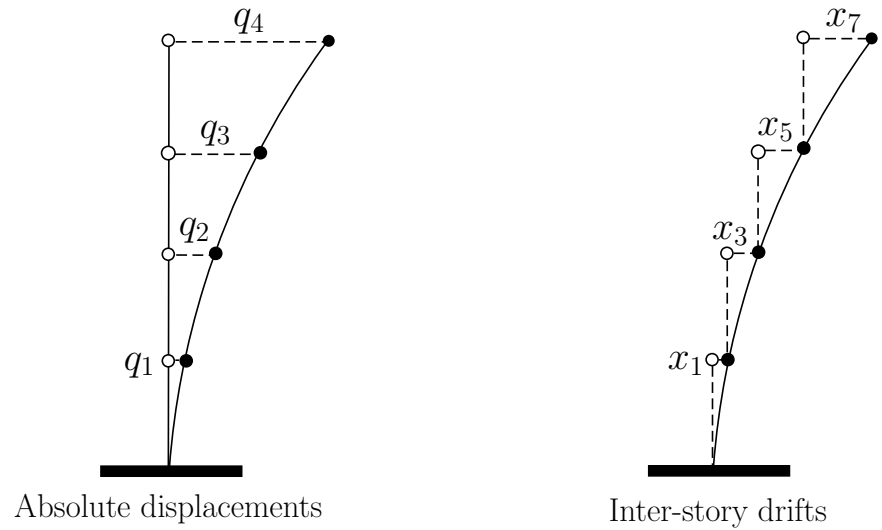

Fig. 6. Absolute and relative displacements.

A detailed derivation of the first-order state-space model can be found in [10]. For the particular values presented in (30), the state and the input matrices are

$$
\begin{aligned}
A & =10^{3} \times\left[\begin{array}{cccccccc}
0 & 0.0010 & 0 & 0 & 0 & 0 & 0 & 0 \\
-0.9850 & -0.0085 & 0.9850 & 0.0085 & 0 & 0 & 0 & 0 \\
0 & 0 & 0 & 0.0010 & 0 & 0 & 0 & 0 \\
0.9850 & 0.0085 & -1.9699 & -0.0170 & 0.9850 & 0.0085 & 0 & 0 \\
0 & 0 & 0 & 0 & 0 & 0.0010 & 0 & 0 \\
0 & 0 & 0.9850 & 0.0085 & -1.9699 & -0.0170 & 0.9850 & 0.0085 \\
0 & 0 & 0 & 0 & 0 & 0 & 0 & 0.0010 \\
0 & 0 & 0 & 0 & 0.9850 & 0.0085 & -1.9699 & -0.0170
\end{array}\right], \\
E & =[0,-1,0,0,0,0,0]^{T},
\end{aligned}
$$

the control matrix for the inter-story actuation scheme is

$$
B^{(a)}=10^{-5} \times\left[\begin{array}{cccc}
0 & 0 & 0 & 0 \\
-0.2894 & 0.2894 & 0 & 0 \\
0 & 0 \\
0.2894 & -0.5787 & 0.2894 & 0 \\
0 & 0 & 0 \\
0 & 0.2894 & -0.5787 & 0.2894 \\
0 & 0 & 0 & 0 \\
0 & 0 & 0.2894 & -0.5787
\end{array}\right]
$$


while the control matrix for the direct actuation scheme is

$$
B^{(b)}=10^{-5} \times\left[\begin{array}{cccc}
0 & 0 & 0 & 0 \\
0.2894 & 0 & 0 & 0 \\
0 & 0 & 0 & 0 \\
-0.2894 & 0.2894 & 0 & 0 \\
0 & 0 & 0 & 0 \\
0 & -0.2894 & 0.2894 & 0 \\
0 & 0 & 0 & 0 \\
0 & 0 & -0.2894 & 0.2894
\end{array}\right] .
$$

\subsection{Centralized controllers}

To compute the centralized optimal LQR controllers, we consider the quadratic index

$$
J_{c}(x(t), u(t))=\int_{0}^{\infty}\left[x^{T}(t) Q^{*} x(t)+u^{T}(t) R^{*} u(t)\right] d t
$$

defined by the weighting matrices $Q^{*}=I_{8}, R^{*}=10^{-15} I_{4}$. Using the Matlab command $\operatorname{lqr}()$ with system matrices $A$ and $B^{(a)}$, we obtain a centralized gain matrix

$$
K_{c}^{(a)}=10^{7} \times\left[\begin{array}{cccccccc}
-0.1466 & -2.8886 & 0 & -0.0048 & 0 & -0.0032 & 0 & -0.0016 \\
0 & -0.0048 & -0.1466 & -2.8870 & 0 & -0.0032 & 0 & -0.0016 \\
0 & -0.0032 & 0 & -0.0032 & -0.1466 & -2.8854 & 0 & -0.0016 \\
0 & -0.0016 & 0 & -0.0016 & 0 & -0.0016 & -0.1466 & -2.8838
\end{array}\right]
$$

for the case of inter-story actuators. The optimal cost $\left[J_{c}^{(a)}\right]_{o p t}=39.58$ is computed as the trace of the matrix $P^{(a)}$, which is obtained as the solution of the corresponding Riccati equation. In the case of direct actuators, the system matrices $A$ and $B^{(b)}$ are used to yield a centralized gain matrix

$$
K_{c}^{(b)}=10^{7} \times\left[\begin{array}{rrrrrrrr}
0.1457 & 2.4536 & -0.0009 & -1.2934 & -0.0006 & -0.3237 & -0.0003 & -0.1065 \\
0.1447 & 1.1602 & 0.1450 & 2.1298 & -0.0012 & -1.3998 & -0.0006 & -0.3237 \\
0.1441 & 0.8365 & 0.1444 & 1.0537 & 0.1450 & 2.1298 & -0.0009 & -1.2934 \\
0.1438 & 0.7300 & 0.1441 & 0.8365 & 0.1447 & 1.1602 & 0.1457 & 2.4536
\end{array}\right]
$$

with an optimal cost $\left[J_{c}^{(b)}\right]_{o p t}=45.06$.

It is worth noting that the gain matrix $K_{c}^{(a)}$, obtained in the case of interstory actuators, exhibits an almost-decentralized structure. This fact can be explained by the particular structure of the physical system and the actuation scheme. In this case, a multi-overlapping controller, or even a decentralized controller, could be obtained by removing some almost-zero elements. The structured controller so obtained is a small perturbation of the optimal controller, and it is reasonable to expect that it will present a good performance. However, it must be emphasized that even in this extremely favorable case, a centralized controller needs to be previously computed in order to apply this strategy. Hence, we would be carrying out a centralized design of a decentralized controller. Of course, this approach cannot be applied to the case of direct actuators, where a full gain matrix $K_{c}^{(b)}$ has been obtained. 


\subsection{Overlapping controllers}

Inter-story actuators. Let us consider the main system $\mathbf{S}^{(a)}$ defined by the pair $\left(A, B^{(a)}\right)$. We see now this system as formed by three subsystems $\mathbf{S}^{(j)}$, corresponding to a block of two consecutive stories of the building. Schematically, we write $\mathbf{S}^{(1)}=[1,2], \mathbf{S}^{(2)}=[2,3], \mathbf{S}^{(3)}=[3,4]$ (see Fig. 7 ). This decomposition

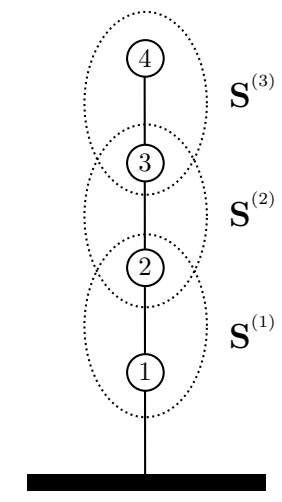

Fig. 7. Multi-overlapping decomposition for a four-story building.

defines a multi-overlapping decomposition with two overlapping blocks corresponding to stories 2 and 3 . The state subsystem dimensions are $d_{x}^{(j)}=4$, $j=1,2,3$, with overlapping blocks of size $o_{x}^{(j)}=2, j=1,2$. Regarding the inputs, the subsystem dimensions are $d_{u}^{(j)}=2, j=1,2,3$, with overlapping blocks of size $o_{u}^{(j)}=1, j=1,2$.

Following the notation introduced in Section 3, we have $r=2$ overlapping blocks and we start with the pair $P_{1}^{(3)}=\left(A, B^{(a)}\right)$. The numbers that define the first expansion step (with index $j=2$ ) are

$$
\left\{\begin{array} { l } 
{ n _ { 1 } ^ { ( 2 ) } = \sum _ { i = 1 } ^ { 2 } ( d _ { x } ^ { ( i ) } - o _ { x } ^ { ( i ) } ) = 4 } \\
{ n _ { 2 } ^ { ( 2 ) } = o _ { x } ^ { ( 2 ) } = 2 } \\
{ n _ { 3 } ^ { ( 2 ) } = d _ { x } ^ { ( 3 ) } - o _ { x } ^ { ( 2 ) } = 2 }
\end{array} \quad \left\{\begin{array}{l}
m_{1}^{(2)}=\sum_{i=1}^{2}\left(d_{u}^{(i)}-o_{u}^{(i)}\right)=2 \\
m_{2}^{(2)}=o_{u}^{(2)}=1 \\
m_{3}^{(2)}=d_{u}^{(3)}-o_{u}^{(2)}=1
\end{array}\right.\right.
$$

Now, we proceed as described in Section 2 and we use the expansion matrices $V^{(2)}, R^{(2)}$, defined by the numbers in (40) to compute the decoupled expanded pairs $P_{1}^{(2)}=\left(\tilde{A}_{1}^{(2)}, \tilde{B}_{1}^{(2)}\right)$ and $P_{2}^{(3)}=\left(\tilde{A}_{2}^{(3)}, \tilde{B}_{2}^{(3)}\right)$, where

$$
\begin{aligned}
& \tilde{A}_{1}^{(2)}=10^{3} \times\left[\begin{array}{cccccc}
0 & 0.0010 & 0 & 0 & 0 & 0 \\
-0.9850 & -0.0085 & 0.9850 & 0.0085 & 0 & 0 \\
0 & 0 & 0 \\
0.9850 & 0.0085 & -1.9699 & 0.0010 & 0 & 0 \\
0 & 0 & 0.9850 & 0.0085 \\
0 & 0 & 0.9850 & 0.0085 & -1.9699 & 0.0010 \\
0 & 0 & 0.0170
\end{array}\right], \\
& \tilde{B}_{1}^{(2)}=10^{-5} \times\left[\begin{array}{ccc}
0.2894 & 0.2894 & 0 \\
0 & 0 \\
0.2894 & -0.5787 & 0.2894 \\
0 & 0 & 0 \\
0 & 0.2894 & -0.5787
\end{array}\right]
\end{aligned}
$$


and

$$
\tilde{A}_{2}^{(3)}=10^{3} \times\left[\begin{array}{cccc}
0 & 0.0010 & 0 & 0 \\
-1.9699 & -0.0170 & 0.9850 & 0.0085 \\
0 & 0 & 0.0010 \\
0.9850 & 0.0085 & -1.9699 & -0.0170
\end{array}\right], \quad \tilde{B}_{2}^{(3)}=10^{-5} \times\left[\begin{array}{cc}
0 & 0 \\
-0.5787 & 0.2894 \\
0 & 0 \\
0.2894 & -0.5787
\end{array}\right] .
$$

The pair $P_{2}^{(3)}=\left(\tilde{A}_{2}^{(3)}, \tilde{B}_{2}^{(3)}\right)$ contains no overlapping blocks, and we choose the quadratic index

$$
J_{2}^{(3)}(x(t), u(t))=\int_{0}^{\infty}\left[x^{T}(t)\left[Q_{2}^{(3)}\right]^{*} x(t)+u^{T}(t)\left[R_{2}^{(3)}\right]^{*} u(t)\right] d t
$$

with weighting matrices $\left[Q_{2}^{(3)}\right]^{*}=I_{n^{(3)}}$ and $\left[R_{2}^{(3)}\right]^{*}=10^{-15} I_{n^{(3)} \times m^{(3)}}$, where $n^{(3)}=$ $n_{2}^{(2)}+n_{3}^{(2)}, m^{(3)}=m_{2}^{(2)}+m_{3}^{(2)}$, to compute the expanded gain matrix

$$
\tilde{K}_{2}^{(3)}=10^{7} \times\left[\begin{array}{cccc}
-0.1466 & -2.8833 & 0 & -0.0005 \\
0 & -0.0005 & -0.1466 & -2.8833
\end{array}\right] .
$$

The expanded pair $P_{1}^{(2)}=\left(\tilde{A}_{1}^{(2)}, \tilde{B}_{1}^{(2)}\right)$ still contains overlapping blocks and it is supplied as the starting point for the next expansion step (with index $j=1$ ), which uses the numbers

$$
\left\{\begin{array} { l } 
{ n _ { 1 } ^ { ( 1 ) } = \sum _ { i = 1 } ^ { 1 } ( d _ { x } ^ { ( i ) } - o _ { x } ^ { ( i ) } ) = 2 } \\
{ n _ { 2 } ^ { ( 1 ) } = o _ { x } ^ { ( 1 ) } = 2 } \\
{ n _ { 3 } ^ { ( 1 ) } = d _ { x } ^ { ( 2 ) } - o _ { x } ^ { ( 1 ) } = 2 }
\end{array} \quad \left\{\begin{array}{l}
m_{1}^{(1)}=\sum_{i=1}^{1}\left(d_{u}^{(i)}-o_{u}^{(i)}\right)=1 \\
m_{2}^{(1)}=o_{u}^{(1)}=1 \\
m_{3}^{(1)}=d_{u}^{(2)}-o_{u}^{(1)}=1
\end{array}\right.\right.
$$

to define expansion matrices $V^{(1)}, R^{(1)}$ in order to produce a new set of decoupled expanded pairs $P_{1}^{(1)}=\left(\tilde{A}_{1}^{(1)}, \tilde{B}_{1}^{(1)}\right)$ and $P_{2}^{(2)}=\left(\tilde{A}_{2}^{(2)}, \tilde{B}_{2}^{(2)}\right)$. The pair $P_{2}^{(2)}=\left(\tilde{A}_{2}^{(2)}, \tilde{B}_{2}^{(2)}\right)$ contains no overlapping blocks and we use a quadratic index $J_{2}^{(2)}$ with weighting matrices $\left[Q_{2}^{(2)}\right]^{*}=I_{n^{(2)}}$ and $\left[R_{2}^{(2)}\right]^{*}=10^{-15} I_{n^{(2)} \times m^{(2)}}$, where $n^{(2)}=n_{2}^{(1)}+n_{3}^{(1)}, m^{(2)}=m_{2}^{(1)}+m_{3}^{(1)}$, to compute the expanded gain matrix

$$
\tilde{K}_{2}^{(2)}=10^{7} \times\left[\begin{array}{cccc}
-0.1466 & -2.8833 & 0 & -0.0005 \\
0 & -0.0005 & -0.1466 & -2.8833
\end{array}\right] \text {. }
$$

As we have completed the expansion process, the expanded pair $P_{1}^{(1)}$ also has no overlapping blocks. We select the quadratic index $J_{1}^{(1)}$ with weighting matrices $\left[Q_{1}^{(1)}\right]^{*}=I_{n^{(1)}}$ and $\left[R_{1}^{(1)}\right]^{*}=10^{-15} I_{n^{(1)} \times m^{(2)}}, n^{(1)}=n_{1}^{(1)}+n_{2}^{(1)}, m^{(1)}=m_{1}^{(1)}+m_{2}^{(1)}$, and compute the expanded gain matrix

$$
\tilde{K}_{1}^{(1)}=10^{7} \times\left[\begin{array}{cccc}
-0.1466 & -2.8854 & 0 & -0.0016 \\
0 & -0.0016 & -0.1466 & -2.8838
\end{array}\right] \text {. }
$$

The contraction process starts with the matrix $\bar{K}^{(1)}=\operatorname{diag}\left(\tilde{K}_{1}^{(1)}, \tilde{K}_{2}^{(2)}\right)$, which 
is contracted to obtain

$$
\tilde{K}_{1}^{(2)}=Q^{(1)} \bar{K}^{(1)} V^{(1)}=10^{7} \times\left[\begin{array}{cccccc}
-0.1466 & -2.8854 & 0 & -0.0016 & 0 & 0 \\
0 & -0.0008 & -0.1466 & -2.8835 & 0 & -0.0003 \\
0 & 0 & 0 & -0.0005 & -0.1466 & -2.8833
\end{array}\right] .
$$

In the second contraction step, the matrix $\bar{K}^{(2)}=\operatorname{diag}\left(\tilde{K}_{1}^{(2)}, \tilde{K}_{2}^{(3)}\right)$ is contracted to obtain

$$
\tilde{K}_{1}^{(3)}=10^{7} \times\left[\begin{array}{cccccccc}
-0.1466 & -2.8854 & 0 & -0.0016 & 0 & 0 & 0 & 0 \\
0 & -0.0008 & -0.1466 & -2.8835 & 0 & -0.0003 & 0 & 0 \\
0 & 0 & 0 & -0.0003 & -0.1466 & -2.8833 & 0 & -0.0003 \\
0 & 0 & 0 & 0 & 0 & -0.0005 & -0.1466 & -2.8833
\end{array}\right] .
$$

This last matrix is the desired multi-overlapping controller, which we denote by $K_{o}^{(a)}$.

Direct actuators. We consider now the main system $\mathbf{S}^{(b)}$ defined by the pair $\left(A, B^{(b)}\right)$. In this case, the previously described procedure yields the expanded gain matrices

$$
\begin{aligned}
\tilde{K}_{1}^{(1)} & =10^{7} \times\left[\begin{array}{rrrr}
0.1463 & 2.5551 & -0.0003 & -1.1441 \\
0.1459 & 1.4110 & 0.1463 & 2.5551
\end{array}\right], \\
\tilde{K}_{2}^{(2)} & =10^{7} \times\left[\begin{array}{rrrr}
2.5977 & 2.2173 & 2.8431 & -1.2756 \\
-5.6100 & 1.2415 & 2.7594 & 2.5171
\end{array}\right], \\
\tilde{K}_{2}^{(3)} & =10^{7} \times\left[\begin{array}{rrrr}
2.5977 & 2.2173 & 2.8431 & -1.2756 \\
-5.6100 & 1.2415 & 2.7594 & 2.5171
\end{array}\right] .
\end{aligned}
$$

After completing the contraction process, the following multi-overlapping controller results

$$
K_{o}^{(b)}=10^{7} \times\left[\begin{array}{cccccccc}
0.1463 & 2.5551 & -0.0003 & -1.1441 & 0 & 0 & 0 & 0 \\
0.0730 & 0.7055 & 1.3720 & 2.3862 & 1.4215 & -0.6378 & 0 & 0 \\
0 & 0 & -2.8050 & 0.6207 & 2.6785 & 2.3672 & 1.4215 & -0.6378 \\
0 & 0 & 0 & 0 & -5.6100 & 1.2415 & 2.7594 & 2.5171
\end{array}\right] .
$$

\section{Numerical simulation}

In this section, the optimal LQR centralized controllers obtained in Subsection 4.2 are taken as a reference to assess the performance of the corresponding multi-overlapping controllers computed through the proposed sequential decomposition procedure. Firstly, for each one of the two considered actuation schemes, the suboptimal cost of the multi-overlapping controllers with respect to the centralized quadratic index $J_{c}$, defined in (37), is computed and compared with the corresponding optimal values. Secondly, numerical simulations of the free response and the controlled response of the system under a seismic excitation are carried out for all the considered controllers. The maximum absolute inter-story drifts, and the maximum absolute control actions are computed and pertinently compared. The 1940 El Centro NS earthquake (Fig. 8), scaled to a peak of $1 \mathrm{~m} / \mathrm{s}^{2}$, has been used as ground acceleration input in the simulations. 


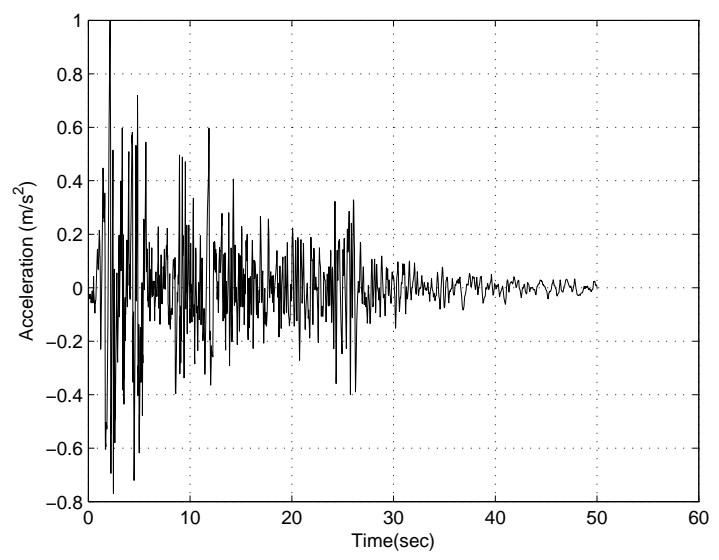

Fig. 8. El Centro NS earthquake, scaled to a peak of $1 \mathrm{~m} / \mathrm{s}^{2}$.

Inter-story actuators. In this case, the centralized optimal LQR gain matrix $K_{c}^{(a)}$ and the multi-overlapping gain $K_{o}^{(a)}$ are very similar:

$$
\begin{aligned}
& K_{c}^{(a)}=10^{7} \times\left[\begin{array}{cccccccc}
-0.1466 & -2.8886 & 0 & -0.0048 & 0 & -0.0032 & 0 & -0.0016 \\
0 & -0.0048 & -0.1466 & -2.8870 & 0 & -0.0032 & 0 & -0.0016 \\
0 & -0.0032 & 0 & -0.0032 & -0.1466 & -2.8854 & 0 & -0.0016 \\
0 & -0.0016 & 0 & -0.0016 & 0 & -0.0016 & -0.1466 & -2.8838
\end{array}\right], \\
& K_{o}^{(a)}=10^{7} \times\left[\begin{array}{cccccccc}
-0.1466 & -2.8854 & 0 & -0.0016 & 0 & 0 & 0 & 0 \\
0 & -0.0008 & -0.1466 & -2.8835 & 0 & -0.0003 & 0 & 0 \\
0 & 0 & 0 & -0.0003 & -0.1466 & -2.8833 & 0 & -0.0003 \\
0 & 0 & 0 & 0 & 0 & -0.0005 & -0.1466 & -2.8833
\end{array}\right] .
\end{aligned}
$$

The cost of the quadratic index $J_{c}$ associated to the controller $u(t)=-K_{o}^{(a)} x(t)$, can be computed as $\left[J_{c}^{(a)}\right]_{o}=\operatorname{trace}\left(P_{o}^{(a)}\right)$, where the matrix $P_{o}^{(a)}$ can be obtained using the Matlab command lyap( ) with arguments $A_{c l}^{T}=\left(A-B^{(a)} K_{o}^{(a)}\right)^{T}$ and $H=Q^{*}+\left[K_{o}^{(a)}\right]^{T} R^{*} K_{o}^{(a)}$. The resulting cost is $\left[J_{c}^{(a)}\right]_{o}=38.58$, which is practically equal to the optimal cost. The upper plot in Fig. 9 displays the maximum (unsigned) inter-story drifts for the uncontrolled system, the optimal LQR centralized controller, and the multi-overlapping controller. In the lower plot, the maximum (unsigned) control efforts are shown. As may be expected from the small differences observed in the gain matrices, the behavior of the optimal LQR centralized controller and the multi-overlapping controller are practically equal; in fact, the corresponding graphs overlap and appear as a single line.

Direct actuators. In this second case, the gain matrices are

$$
\begin{gathered}
K_{c}^{(b)}=10^{7} \times\left[\begin{array}{rrrrrrrr}
0.1457 & 2.4536 & -0.0009 & -1.2934 & -0.0006 & -0.3237 & -0.0003 & -0.1065 \\
0.147 & 1.1602 & 0.1450 & 2.1298 & -0.0012 & -1.3998 & -0.0006 & -0.3237 \\
0.1441 & 0.8365 & 0.1444 & 1.0537 & 0.1450 & 2.1298 & -0.0009 & -1.2934 \\
0.1438 & 0.7300 & 0.1441 & 0.8365 & 0.1447 & 1.1602 & 0.1457 & 2.4536
\end{array}\right], \\
K_{o}^{(b)}=10^{7} \times\left[\begin{array}{cccccccc}
0.1463 & 2.5551 & -0.0003 & -1.1441 & 0 & 0 & 0 & 0 \\
0.0730 & 0.7055 & 1.3720 & 2.3862 & 1.4215 & -0.6378 & 0 & 0 \\
0 & 0 & -2.8050 & 0.6207 & 2.6755 & 2.3672 & 1.4215 & -0.6378 \\
0 & 0 & 0 & 0 & -5.6100 & 1.2415 & 2.7594 & 2.5171
\end{array}\right]
\end{gathered}
$$



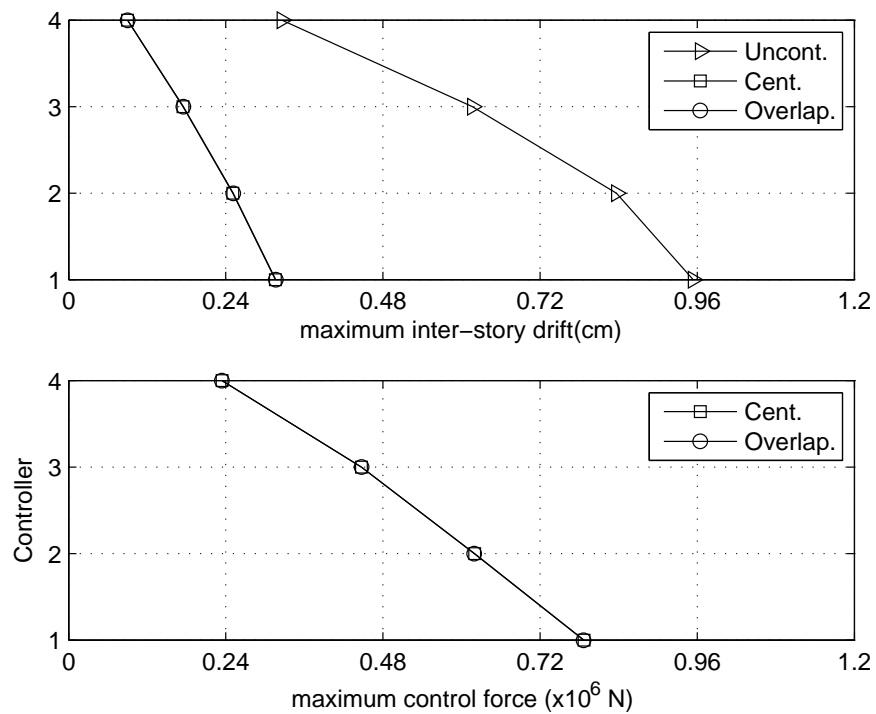

Fig. 9. Maximum inter-story drifts and control forces for inter-story actuators.

The centralized controller has an optimal cost $\left[J_{c}^{(b)}\right]_{\text {opt }}=45.06$, while the multioverlapping controller has a suboptimal cost $\left[J_{c}^{(a)}\right]_{o}=46.99$. The maximum inter-story drifts and maximum control efforts are displayed in Fig. 10.
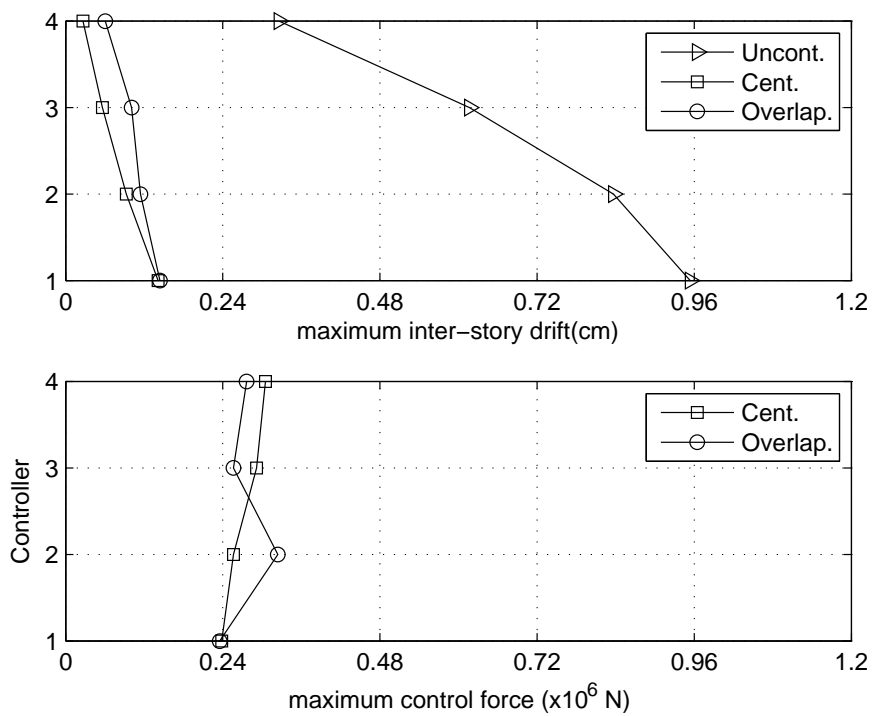

Fig. 10. Maximum inter-story drifts and control forces for direct actuators.

It can be observed that, even in this case where the optimal centralized gain matrix is a full matrix, the multi-overlapping controller maintains a remarkable high-level of performance. 


\section{Conclusions}

In this paper a sequential procedure to design multi-overlapping controllers for systems admitting a longitudinal multi-overlapping decomposition has been presented. By means of the proposed methodology, the design of a multi-overlapping controller can be reduced to the design of a series of lowerdimension independent controllers. To illustrate the application of the proposed decentralized design strategy, a control system to mitigate the vibrational response of a building under seismic excitation has been chosen. More precisely, multi-overlapping controllers have been designed for a simplified onedimensional model of a four-story building with two actuation schemes. Numerical simulations of the controlled building response indicate a remarkably good behavior of the designed multi-overlapping controllers when compared with the corresponding optimal LQR centralized controllers, which has been taken as a reference.

\section{References}

[1] L. Bakule, J. Rodellar, and J.M. Rossell. Generalized selection of complementary matrices in the inclusion principle. IEEE Transactions on Automatic Control, 45(6):1237-1243, 2000.

[2] L. Bakule, J. Rodellar, and J.M. Rossell. Structure of expansion-contraction matrices in the inclusion principle for dynamic systems. SIAM Journal on Matrix Analysis and Applications, 21(4):1136-1155, 2000.

[3] L. Bakule, J. Rodellar, and J.M. Rossell. Controllability-observability of expanded composite systems. Linear Algebra and its Applications, 332-334:381400, 2001.

[4] L. Bakule, J. Rodellar, J.M. Rossell, and P. Rubió. Preservation of controllability-observability in expanded systems. IEEE Transactions on Automatic Control, 46(7):1155-1162, 2001.

[5] X.-B. Chen and S.S. Stanković. Decomposition and decentralized control of systems with multi-overlapping structure. Automatica, 41:1765-1772, 2005.

[6] A. İftar and Ü. Özgüner. Contractible controller design and optimal control with state and input inclusion. Automatica, 26(3):593-597, 1990.

[7] M. Ikeda and D.D. Šiljak. Overlapping decentralized control with input, state, and output inclusion. Control-Theory and Advanced Technology, 2(2):155-172, 1986.

[8] S.S. Stanković and D.D. Šiljak. Contractibility of overlapping decentralized control. Systems \& Control Letters, 44:189-199, 2001. 
[9] D.D. Šiljak. Decentralized Control of Complex Systems. Academic Press, New York, USA, 1991.

[10] Y. Wang, J.P. Lynch, and K.H. Law. Decentralized $H_{\infty}$ controller design for large-scale civil structures. Earthquake Engineering and Structural Dynamics, $38: 377-401,2009$. 